\title{
El liderazgo transformacional y su correlación con el bienestar psicológico en el trabajo en las MiPyMes industriales de la ciudad de Durango México
}

\author{
Dra. María Brenda González Herrera \\ brenda.gonzalez@ujed.mx \\ https://orcid.org/0000-0003-3697-2074 \\ Universidad Juárez del Estado de Durango \\ Dr. José Gerardo Ignacio Gómez Romero \\ gerardoignaciog@yahoo.com.mx \\ https://orcid.org/0000-0002-6322-6133 \\ Universidad Juárez del Estado de Durango \\ Dr. Ernesto Geovani Figueroa González \\ geovani.figueroa@ujed.mx \\ https://orcid.org/0000-0002-7900-9141 \\ Universidad Juárez del Estado de Durango \\ Dr. Rosalío Tortolero Portugal \\ chalioby@hotmail.com \\ https://orcid.org/0000-0002-4526-7417 \\ Centro Educativo de Líderes Durango
}

\section{RESUMEN}

El propósito de la presente investigación es determinar la relación que existe entre el liderazgo transformacional y el bienestar psicológico en el trabajo percibido por los trabajadores de las MiPyMes industriales de la ciudad de Durango México. Para la recopilación de la información se utilizó la técnica de la encuesta. El instrumento de medición se conforma por 36 reactivos en escala Likert y se aplicó en el segundo semestre del año 2019 a 462 trabajadores pertenecientes a 337 empresas estudiadas. Se utilizo la técnica de análisis estadístico de regresión lineal simple, se revisó la validez y el ajuste del modelo; se propuso una ecuación de regresión y se realizó el análisis de los supuestos. Se concluye que la correlación entre ambas variables es positiva y el modelo de regresión lineal cumple con los supuestos.

Palabras clave: bienestar psicológico; liderazgo transformacional; regresión lineal. 


\title{
Transformational leadership and its correlation with psychological well-being at work in industrial MIPYMES in the city of Durango, Mexico
}

\begin{abstract}
The purpose of this research is to determine the relationship between transformational leadership and psychological well-being at work perceived by workers of industrial MSMEs in the city of Durango, Mexico. The survey technique was used to collect the information. The measurement instrument is made up of 36 items on the Likert scale and was applied in the second half of 2019 to 462 workers belonging to 337 companies studied. The simple linear regression statistical analysis technique was used, the validity and fit of the model was reviewed; A regression equation was proposed and the analysis of the assumptions was carried out. It is concluded that the correlation between both variables is positive and the linear regression model complies with the assumptions.
\end{abstract}

Keywords: psychological well-being; transformational leadership; linear regression

Artículo recibido: 10 Setiembre. 2021 Aceptado para publicación: 15 Octubre. 2021 Correspondencia: brenda.gonzalez@ ujed.mx Conflictos de Interés: Ninguna que declarar 


\section{INTRODUCCIÓN}

El bienestar laboral, tradicionalmente se ha considerado como un conjunto de iniciativas dirigidas a mejorar las condiciones de los trabajadores dentro de sus organizaciones. En el contexto nacional mexicano, donde los ciudadanos se desenvuelven en una economía emergente, el bienestar en los ámbitos laborales representa dos cosas; una fuente de mejora en la calidad de vida de la población, y el desarrollo psicológico y motivado que le permita ejercer de forma óptima sus actividades laborales, contribuyendo así a integrar una sociedad más preparada y competitiva que genere una economía más estable a nivel local, nacional y global.

Ante tal panorama, los líderes de las organizaciones buscan desesperadamente encontrar una fórmula cortoplacista que dé solución a sus problemas inmediatos generalmente de tipo financiero, sin darse cuenta que la búsqueda de su solución debe ser integral, por un lado, buscando ser innovadores pero por otro, involucrar en la toma participativa de decisiones y permitir a su personal tomar parte en la resolución proactiva del problema, logrando con esto incrementar su rendimiento y el logro de objetivos organizacionales.

Sin embargo, esto no sucederá si no se deja de ver al ser humano solo como un elemento o factor de la producción, que puede ser reemplazado y objetivizado a capricho de quien toma las decisiones en una empresa. El enfoque del líder debe cambiar construyendo y dirigiendo organizaciones más humanas, en donde sea importante el cuidado del Bienestar Psicológico en el Trabajo (BPT), pues de esto depende su rendimiento y compromiso.

Uno de los rasgos de la problemática, exige la definición de políticas de gestión del talento humano acordes a las nuevas demandas, para mantener la capacidad competitiva de las organizaciones en cuanto a conceptos de eficiencia, eficacia y calidad. En este contexto, en muchas ocasiones se pierde de vista que el personal es mucho más que un instrumento, y su gestión, debe considerar que las personas son el capital más importante de una empresa. Éste posee características y habilidades propias que dotan de identidad, movimiento y vida a toda la organización, si el elemento humano está dispuesto a aportar su esfuerzo, conocimiento y habilidades, la organización funcionará y se nutrirá de su capital humano, el cual podrá representar incluso un mecanismo de mejora continua. De lo contrario, la organización se estancará y estará predestinada al fracaso. 
Aunado a ello, la hipótesis que defiende que "el trabajador feliz y con bienestar es más productivo" cuestionada por Peiró, Tordera, Lorente, Rodríguez, y Ayala, (2014), arroja datos vagos o poco precisos de la correlación que existe entre dichas variables y, en su caso, los datos que demuestran una correlación certera, suelen pertenecer a estudios realizados por compañías con estructuras sólidas, y programas que dedican gran cantidad de recursos a investigar y mejorar las condiciones laborales, a la par que dan seguimiento a su estilo de dirección y sus planes estratégicos, lo cual representa el menor porcentaje de empresas (grandes empresas y transnacionales).

En gran medida en México, la Ley Federal del Trabajo a través de la Secretaría del Trabajo y Previsión Social (STPS) faculta a la autoridad para asegurar que las fuentes de trabajo cubran las necesidades vitales y psicológicas de los empleados, siendo el caso de la reciente puesta en marcha NOM-35, así como el acceso a la salud y a otras necesidades básicas, como son el derecho a la vivienda y a el vestido. Sin embargo existe poca evidencia del estudio del buen clima organizacional para regular cargas excesivas de trabajo, o el trato no adecuado por parte de los gerentes hacia los trabajadores, por ende, toma relevancia considerar la importancia de resaltar factores como la influencia del líder de la organización con necesidades psicológicas de los empleados como el equilibrio vida-trabajo, escenarios de desarrollo personal y plenitud al integrar el del aspecto laboral a su propio proyecto de vida, es una cuestión que hasta hace poco ha comenzado a estudiarse para relacionarla con las estrategias organizacionales formales.

Existen estudios relativamente recientes que enfatizan la relación de la felicidad en los seres humanos y el efecto que éste produce en el trabajo, encontrándose en algunos casos que una variable afecta a la otra de manera significativa. Se encuentra evidencia a través de indicadores de correlación, que las personas felices aprenden mejor, y que las emociones y pensamientos negativos afectan la atención y el rendimiento, en contraparte, las emociones positivas generan un pensamiento creativo y holístico, que se relaciona con la innovación, así como mayor desempeño y atención en las tareas (Diener y BiswasDiener, 2002; Diener y Seligman, 2004; Cropanzano y Wright, 2001; Bolte, Goschke y Kuhl, 2003; Kuhl, 2000; Seligman, Gillham, Reivich y Linkins, 2009).

Se ha puesto en evidencia que la felicidad no constituye solo aspectos afectivos del ser humano, toda vez que deben considerarse también elementos cognitivos y la importancia que tiene para las personas definir el sentido de la vida y los factores necesarios para su 
crecimiento personal. Derivado de esto, se ha ampliado él término felicidad del ser humano al de bienestar.

Por tanto, la dirección que se recibe en una organización tiene un rol primordial para alcanzar el bienestar psicológico. Sin embargo, la tendencia empresarial y la toma de decisiones de los líderes se orienta hacia la reducción indiscriminada de costos, lo que incide en el creciente deterioro del bienestar de los trabajadores, ocasionando bajo nivel de productividad y competitividad (Peiró et al. 2014).

La ponderación de las variables descritas pone de manifiesto el papel del líder en la adecuada gestión de las empresas. Es necesario contar con un liderazgo que redimensione el papel del bienestar en la gestión de talento, como aliado estratégico de la productividad, el compromiso y el éxito empresarial.

Lo anteriormente descrito se potencializa cuándo se enfoca el análisis a las MiPyMes (micro, pequeñas y medianas empresas), las cuales a nivel mundial su expansión ha ido en aumento. En Latinoamérica y específicamente en México, estas empresas se han posicionado durante los últimos decenios como importantes motores de la economía, al generar de manera significativa empleo y autoempleo. De los 4.9 millones de establecimientos del sector privado y paraestatal registrados en los Censos Económicos 2019, 99.8\% pertenecen a las MiPyMes que en conjunto aportan el 52\% del Producto Interno Bruto (PIB), de acuerdo con datos del Instituto Nacional de Estadística y Geografía (INEGI), actualizados a 2021.

Por su parte, Avendaño y Perrotini (2015), basándose en datos del Banco de México y del Banco Mundial, mencionan que a causa de fallas del mercado y de gobierno, así como de diversas variables económicas y financieras, México ha tenido un lento crecimiento, sino es que estancado, desde 1980. Dichos factores limitan las posibilidades de acción de los micro y pequeños empresarios en cuanto a elevar el potencial de sus recursos financieros y materiales, excepto con respecto al capital humano, que es un recurso de primer orden para impulsar la productividad, competitividad e incluso la rentabilidad de sus empresas, y para evitar su desaparición en el primero o segundo año de vida.

En cuanto a las carencias en la teoría y la práctica, según Vázquez (2018), se pueden citar las siguientes:

- Falta de definición de las variables más significativas en lo socio psicológico, que pueden influir en los avances de las MiPyMes. 
- En Latinoamérica y México, aunque hay trabajo teórico de investigación, las aplicaciones en la empresa tienen pocos registros y escasa información sobre las consideraciones de dichos aspectos.

- Solo pocas organizaciones, en su mayoría de gran tamaño, están incursionando en inversiones relacionadas con gestión de talento enfocada en el concepto de bienestar o un mejor desempeño, lo que deja a las MiPyMes como un sector desconocido para su aplicación.

Como se menciona en los reportes de Great Place to Work edición 2020, en los informes de Manpower (2020), incluso en los de Grupo Deloitte México (2019), los mejores lugares de trabajo, que aumentan su competitividad, permanencia y éxito son aquéllos que se esfuerzan en ofrecer mejores condiciones laborales. Pero, sobre todo, son aquellos en donde el líder o directivo invierte en aspectos positivos como el balance de vida, ética y responsabilidad social, buenas relaciones laborales (compañerismo), confianza, credibilidad de la empresa, respeto y equidad, así como el sentido de pertenencia que puede ser un generador de compromiso, trascendencia y emociones positivas, variables explicitas del concepto de bienestar psicológico.

De ahí la importancia de estudiar la variable del estilo de liderazgo y su correlación con el BPT, partiendo del análisis de las observaciones realizadas por organismos como la Comisión Económica para América Latina y el Caribe (CEPAL, 2019), y la Organización para la Cooperación y el Desarrollo Económico (OCDE, 2020), a los cuales instituciones como la Universidad Nacional Autónoma de México (UNAM) se han sumado y considera de gran relevancia en el estudio académico e investigativo, ya que, además de acabar con prejuicios y desconocimiento sobre el tema, impulsa a la investigación con resultados efectivos que constituyan un parámetro para replantear políticas públicas que generen condiciones para que los individuos alcancen niveles mayores de bienestar psicológico de forma sostenida promovida por sus líderes (Millán y Castellanos, 2018).

Sobre esto, Delfín y Acosta (2016) acotan que, cuando una organización alcanza un óptimo desarrollo organizacional, permite que a los gerentes y dueños de las empresas se les presente un sin número de mejoras en su medio empresarial; siendo el reconocimiento de las capacidades con que la organización cuenta una de las herramientas fundamentales. El líder será el responsable de aprovechar o desperdiciar estas ventajas. Es por ello que se le ha dado un enfoque especial al estilo de liderazgo, su importancia y su función en la 
dinámica de las empresas, ya que el líder es quien podría tomar las decisiones importantes sobre el funcionamiento de la organización.

Para ahondar en este tema, es preciso señalar que el concepto de liderazgo es, de acuerdo con García, Salas-Arbeláez y Gaviria (2017), un proceso natural de influencia entre una persona (el líder) y sus seguidores, el cual puede ser explicado a través de ciertas características y conductas del líder, por percepciones y atribuciones del seguidor o por el contexto en el que ocurre la presencia del liderazgo.

Se podría identificar que tiene su origen histórico en el momento en que el hombre comenzó a organizarse en grupos para modificar las condiciones de vida por necesidades de supervivencia (Izquierdo, Novillo, y Mocha, 2017). Como Ferrer (2015) determina, existen tantas teorías de liderazgo como prácticas de gestión de recursos humanos orientados en ellas, los cuales, son dos importantes vías de interacción entre la organización y su personal.

Independientemente de que una empresa u organización cuente con un equipo de trabajo talentoso y comprometido, siempre será necesario un líder capaz de orientar y coordinar los esfuerzos y dirigir el trabajo del equipo al logro de los objetivos propuestos, como menciona Cújar, Ramos, Hernández, y López (2014), en el ámbito organizacional, García et al, (2017), destaca que el gerente que es líder trabaja para ser aceptado (utilizando para ello su carisma y servicio) por un equipo que requiere de su ayuda y orientación para cumplir con las metas de la organización.

El liderazgo gerencial efectivo debe fomentar la responsabilidad, el espíritu de equipo, el desarrollo personal, y, especialmente, promover el sentido de pertenencia que une a los trabajadores para decidir las líneas de acción que conduzcan al cumplimiento de objetivos y metas.

Por ello, es primordial la implantación de un modelo de liderazgo de alta efectividad dentro de una organización, entendida como un sistema orientado a resultados mediante la integración del talento humano con los recursos financieros, técnicos y materiales. Liderar una organización representa retos, que se van formando día a día, donde no es lo mismo que liderar a un grupo. Por ello, es importante tener líderes capaces de posicionar a la organización y no jefes en el sentido tradicional reacios a implementar cambios (García et al. 2017). 
Se han estudiado alrededor de 65 clasificaciones de los estilos de liderazgo, durante los últimos 50 años, todos ellos coinciden en cuatro características o componentes centrales, los cuales son; el liderazgo como un proceso, tiene que ver con la influencia, ocurre dentro de un grupo y busca conseguir resultados, según Castro (2010), además se destaca que los principales enfoques teóricos acerca del liderazgo son:

a) El enfoque de los Rasgos,

b) El enfoque conductual,

c) El enfoque situacional,

d) El enfoque de Bass sobre el Liderazgo Transformacional y Transaccional y,

e) Otros enfoques actuales.

Para efectos de la presente investigación, se considera relevante el enfoque transformacional de Bass (1985), en el cual los seguidores trascienden sus propias necesidades, los líderes se enfocan en los intereses y necesidades de desarrollo de sus seguidores con el fin de cumplir las metas del grupo (García et al. 2017).

Por otro lado, Bass (1985) señala que, el líder transformacional o el estilo de liderazgo transformacional logra que los seguidores estén conscientes de las necesidades personales de crecimiento, de desarrollo y realización.

Para Perilla y Gómez (2017), el tipo de liderazgo a considerar para propiciar el BPT es el transformacional, debido a la importancia que el líder les da a las necesidades individuales de sus seguidores siendo el medio de obtener los resultados y metas organizacionales, Lapo y Jácome (2015) respecto al liderazgo transformacional determinan que es la capacidad de conducir a las personas de una organización para alcanzar los objetivos de ésta. Para ello, la dirección deberá de estar basada en el respeto al líder generado por sus cualidades profesionales y personales; así como por sus conocimientos y capacidad para transmitirlos.

Otra particularidad que Bass identifica en los líderes transformacionales es que a través de la influencia ejercida en sus sinergias con los miembros del grupo estimulan cambios de visión que conducen a cada individuo a dejar de lado sus intereses particulares para buscar el interés colectivo, incluso cuando el subordinado no tenga satisfechas sus necesidades vitales existenciales (Valderrama, 2018).

Carol (2017) plantea las ventajas del liderazgo transformacional en el equipo de trabajo; y destaca que el liderazgo transformacional será congruente con el plan estratégico de la 
empresa constituyendo de esta forma una herramienta para lograr los objetivos de la empresa, lo que generará ventajas para la organización. Es por ello que:

- Busca inspirar a sus colaboradores dando significado a sus actividades y haciendo propia la meta por alcanzar, a la vez que los impulsa a desempeñarse mejor fomentando su desarrollo personal.

- Fomentar el sentido de corresponsabilidad entre los miembros del equipo dándoles libertad para hacer su trabajo, pero al mismo tiempo haciéndoles propietarios y responsables del mismo. Esto generará la retención de empleados y clientes, disminuyendo así los costos inherentes de la rotación (sinergia sostenible).

- Se involucra completamente con la gente, buscando satisfacer sus necesidades junto con las de la organización, por lo cual es más probable que los empleados se sientan identificados con el líder dando como respuesta su esfuerzo en el trabajo y se queden en la compañía.

Es así como tiene sentido dirigir una organización en pro del bienestar psicológico en el trabajo con vías a fortalecer los objetivos organizacionales.

Lograr los resultados esperados en una organización suele considerarse un proceso difícil, pero mucho más difícil, resulta determinar si los trabajadores sienten satisfacción por alcanzar dichos objetivos y por su contribución para que eso suceda, al margen de que no se puede separar la idea que se tiene por parte de la mayoría de gerencias o jefes, como entendido que así es al recibir un salario que aporte tranquilidad personal y de la familia. Lo anterior es algo que cobra mucha más importancia en los momentos actuales en que no sólo se diversifica la producción, sino también se eleva la complejidad del mundo en que vivimos. El mundo complejo, a decir de Edgar Morín (1994), identifica la realidad y la matiza. En este sentido, es ya imposible pensar en una empresa que deje a un lado a sus trabajadores como personas para contemplarlos como únicamente medios de producción, de ahí la urgencia e importancia de la búsqueda del bienestar en los entornos laborales.

Por otra parte, otro factor que ha influido de forma importante en el auge que ha adquirido el concepto del Bienestar en los entornos laborales ha sido su inclusión en la Declaración Constitucional de la Organización Mundial de la Salud (OMS) de 1948, ya que se utiliza como concepto base para la definición de salud al definirla como "estado de completo bienestar físico, psicológico y social” (Blanch et al. 2010, p. 57). 
Esto ha generado la preocupación a nivel global por fomentar, evaluar y monitorear este concepto tanto a nivel personal como en lo social y, sobre todo, ha llevado a las empresas a interesarse en todo lo referente al bienestar en los entornos de trabajo. Respecto a esto, Blanch et al. (2010) en su investigación Questionnaire of General LaborWell-Being: Structure and Psychometric Properties (Cuestionario general del bienestar laboral: Estructura y propiedades psicométricas), refieren como "las complejas e intensas relaciones entre bienestar y trabajo han centrado el interés de la psicología en general y, particularmente, el de la psicología del trabajo y de las organizaciones" (p. 57).

Se puede concluir, entonces, que la promoción del bienestar en los entornos laborales representa una estrategia de salud para elevar la calidad de vida. Para ello, una evaluación psicológica en los ámbitos laborales deberá analizar los aspectos positivos del funcionamiento de los individuos, es decir, el bienestar psicológico. Esta conclusión se basa en la evidencia empírica de que, diferentes medidas de bienestar psicológico (por ejemplo, afecto positivo y nivel de compromiso) van más allá de evaluar aspectos contrarios de medidas preventivas (por ejemplo, afecto negativo, agotamiento) (Stone y Mackie, 2013).

Sin embargo, los esfuerzos para operacional izar el bienestar psicológico se definen a menudo como la ausencia de alguna patología mental. Esta perspectiva integra los dos enfoques teóricos tomados para conceptualizar y medir el bienestar psicológico; el hedónico (emociones positivas) y eudaimónico (evaluativa). Si se transponlan estos enfoques a los ámbitos laborales, en el enfoque hedónico, el bienestar psicológico es impulsado por una búsqueda de recompensas, placer y la supresión de experiencias negativas, y ocurre cuando se tiene un alto nivel de afecto positivo, y un bajo nivel de negativo, así como un alto nivel de satisfacción con la vida. Por otra parte, el enfoque eudaimónico reflejará la presencia de significados, objetivos y metas en la vida del sujeto a corto y largo plazo (Mosrin, Boudriás, y Marsh, 2017).

Más allá de dividir y determinar qué visión sobre el bienestar psicológico es más aceptada para su investigación, es importante identificar sobre qué aspectos de la satisfacción en la vida de las personas se pretende evaluar. Ambos son válidos, incluso pudiera afirmarse que la conceptualización eudaimónica o evaluativa comprende a su vez la visión hedónica o de los afectos en alguna de sus dimensiones, todo dependerá en tener claro si el estudio 
se dirigirá en obtener indicadores de felicidad (hedónica) o factores relativos a evaluar el desarrollo del potencial humano (eudaimónico) (Reyes, et al., 2016).

A las empresas sobre todo les interesa atender el tema relacionado con la salud tanto mental como física y el bienestar, debido a que existen alarmantes estadísticas que resaltan el problema con los costos relacionados a la atención de enfermedades cada vez más recurrentes como la depresión, trastornos digestivos asociados al estrés, días de trabajo perdidos (ausentismo), presentándose además que la satisfacción en el área de trabajo va en detrimento en los últimos años, hecho que a las empresas de todo tipo les está costando grandes cantidades de dinero atender (Dagenais-Desmarais y Savoie, 2012). Además, dicho conflicto conlleva a su vez el problema de pretender elaborar los estudios bajo un enfoque utilizando un elemento o un solo domino para tratar de explicar todo el bienestar psicológico, siendo que los modelos que trabajan cualquier enfoque antes presentado están fundamentados en una totalidad o una percepción general de la felicidad, la satisfacción o la plenitud del ser humano (Dagenais-Desmarais y Savoie, 2012).

Respecto a lo anterior, Blanch et al. (2010) mencionan que el estado de bienestar laboral es tratado generalmente como consecuencia personal de algún input organizacional, y excepcionalmente como factor causante de determinadas consecuencias personales $\mathrm{u}$ organizacionales. Respaldando las aportaciones de Dagenais-Desmarais y Savoie (2012), mencionan que el común denominador de las escalas aplicadas a investigaciones relacionadas con el bienestar en el trabajo consiste en que fueron realizadas para obtener una medida del bienestar o la salud en general, por lo que sus resultados si bien generan aportes importantes a las consideraciones de las variables, no tendrán la objetividad requerida para generalizar los hallazgos.

No es que los resultados carezcan de un rigor científico y no aporten contenido a la ciencia, la critica que se realiza al respecto es que, aunque se utilizan con frecuencia los modelos, las escalas y medidas del bienestar subjetivo psicológico, parecen estar adaptadas a varios ámbitos o dominios para reflejar adecuadamente una realidad organizativa (especifica), ya que de hecho el trabajo es un dominio distinto de otros (Desmarais-Dagenais y Savoie, 2012).

Como Morin (2004) lo identifica, el trabajo es el único dominio de la vida que proporciona el sustento para lograr las condiciones de una vida digna, por lo que una persona económicamente activa pasa al menos la mitad de su día inmersa en su ambiente. 
Por esta razón se vuelve muy interesante estudiar el concepto de BPT para muchos investigadores, ya que por el papel tan importante que representa en la vida, impacta de alguna manera en los otros dominios como la familia, los amigos, etc.

Bajo esas premisas se han realizado estudios considerables referentes a las determinantes del bienestar psicológico en el trabajo para una mejor gestión organizacional en diferentes tipos de empresas. Los resultados que han arrojado tienen en común que definitivamente se presentan indicadores relacionados con mejores respuestas de los trabajadores correspondientes en mayores niveles de bienestar, pero no se pueden generalizar dichas aportaciones debido a que la referencia teórica está muy focalizada para el área específica de estudio a la vez que las mediciones se obtienen de modelos realizados para campos completos del bienestar y no solamente para el dominio del trabajo (Hindrichs, JuárezGarcía, Girardo y Converso, 2016; Devonish, 2013; Greenidge, Devonish y Alleyne, 2014; Teoh, Coyne, Devonish, Leather y Zarola, 2016; Rahman, Amran, Ahmad y Taghizadeh, 2016; D’Raven y Pasha-Zaidi, 2016).

De lo anteriormente descrito surge la inquietud de realizar un estudio que conteste las preguntas de investigación:

- ¿Existe correlación significativa entre la variable liderazgo transformacional y la variable bienestar psicológica en el trabajo (BPT)?

- ¿El análisis del liderazgo transformacional y el bienestar psicológico en el trabajo cumple con los supuestos del modelo de regresión lineal simple?

\section{ESTRATEGIAS METODOLÓGICAS O MATERIALES Y MÉTODOS}

La investigación es de tipo no experimental, transversal en cuanto su temporalidad y es correlacional para analizar cómo se presenta el grado de asociación entre las variables del estudio y determinar la incidencia de una con la otra. La recopilación de la información fue a través de la técnica de la encuesta, la cual se aplicó en el segundo semestre del año 2019 a 462 trabajadores pertenecientes a 337 empresas estudiadas. Para determinar la relación que existe entre las variables de estudios, con el fin de modelar y predecir el comportamiento de la variable dependiente respecto a la variable independiente, se utilizó la técnica estadística de análisis de regresión lineal simple.

El instrumento de medición utilizado para medir el bienestar psicológico en el trabajo fue diseñado y validado por Dagenais-Desmarais y Savoie (2012), se conforma de 25 ítems en escala Likert ( 1 como nada o nulo, 2 solo un poco, 3 medianamente, 4 pleno/a y 5 
totalmente pleno/a.) e incluye cinco dimensiones significativas las cuales son; ajuste interpersonal en el trabajo, prosperidad laboral, sentimiento de competencia en el trabajo, reconocimiento percibido y deseo de participación en el trabajo. Por su parte, la medición del liderazgo transformacional se realizó por medio de 11 ítems en escala Likert (1 totalmente en desacuerdo, 2 poco de acuerdo, 3 medianamente de acuerdo, 4 de acuerdo, 5 totalmente de acuerdo) a través de la adaptación del instrumento de Cook y Wall (1980). Únicamente se adaptaron los ítems que son referentes a la evaluación de la gerencia por parte de los trabajadores. Para medir el estilo de liderazgo trasformador se empleó el instrumento de Podsakoff, MacKenzie, Moorman, y Fetter, (1990).

El objetivo de la regresión lineal simple es obtener una ecuación válida de la forma;

$$
\mathbf{y}=\mathbf{b 0}+\mathbf{b 1 x}
$$

\section{Donde:}

- y es la variable dependiente

- x es la variable independiente

- b0 es la ordenada al origen

- b1 es la pendiente de la recta

Para realizar un análisis de regresión se tomaron en cuenta los siguientes tres aspectos:

\section{1.- Validez y ajuste del modelo.}

Para que un modelo sea válido debe de cumplirse que $b 1 \neq 0$, ya que si $b 1=0$ entonces la variable x no aportaría nada al modelo, este quedaría y $=b 0$, por lo que no tendría sentido hablar de un modelo en el que y depende de $\mathrm{x}$. Para verificar si $\mathrm{b} 1 \neq 0$, se realizó una prueba de hipótesis que podemos ver en la tabla ANOVA, donde la hipótesis nula es que $b 1=0$, la cual pretendemos rechazar, por lo que se espera que su valor $p$ sea muy bajo. Si se rechaza la hipótesis nula, el modelo tiene sentido, y se concluye que es válido. ¿Qué tan bueno sería el modelo? El ajuste responde a esta pregunta con los coeficientes R, R 2 y R ajustada, $-1 \leq R \leq 1,0 \leq R 2 \leq 1$ y $0 \leq$ Rajustada $\leq 1$. Se suelen utilizar R 2 y $\mathrm{R}$ ajustada corregida para el ajuste del modelo, si son cercanos a 1, el modelo tiene un ajuste muy bueno, y si es cercano a cero, entonces es malo.

\section{2.- Ecuación de regresión.}

Una vez aceptado que el modelo es válido y tiene el suficiente ajuste que se necesita, se procede a revisar los coeficientes b0 y b1, para obtener el modelo. En este sentido, para 
poder generar un modelo de regresión, los datos necesitan cumplir con una serie de condiciones, sin las cuales, no tiene sentido realizar el modelo.

\section{Análisis de los supuestos}

Hay cuatro supuestos que se deben de cumplir: Linealidad, Independencia, Normalidad, Homocedasticidad.

\section{- Linealidad}

Para verificar este supuesto podemos realizar una prueba de correlación lineal previa para verificar si tiene sentido aplicar una regresión lineal, pero si ya empezamos con la prueba, nos podemos fijar en el coeficiente R (correlación de Pearson), R debe cumplir con ser cercano a $10-1$.

\section{- Independencia}

Debemos de verificar que las observaciones muestrales son independientes. Si no se cumple, los estimadores y por lo tanto las predicciones pierden eficacia, y el contraste en la tabla ANOVA no es confiable. Para verificar este supuesto, nos fijamos en el estadístico d de Durbin-Watson, $0<\mathrm{d}<4$. (d debe ser cercano a 2 para concluir que sí hay independencia), si es cercano a 4 o a 0 entonces no la hay, se recomienda que d esté entre 1.5 y 2.5

\section{- Normalidad}

Este supuesto se refiere a que los errores o residuales del modelo sigan una distribución normal. Si no se cumple esta hipótesis los parámetros dejan de ser los máximos verosímiles, y pierden eficacia, pero si la muestra es grande, no es necesario que se cumpla este supuesto con todo rigor. Usaremos el histograma y el gráfico P-P para que realice el contraste de los residuales con la distribución normal. En caso de que la muestra sea grande, en el histograma las barras deben de asemejarse a la curva normal, y en el gráfico P-P los datos deben asemejarse a la línea de $45^{\circ}$.

\section{- Homocedasticidad}

El último supuesto es el de la homocedasticidad de los residuos, es decir, que los residuos tengan varianzas iguales, si no se cumple decimos que hay heterocedasticidad y los parámetros pierden la propiedad de generar un modelo con varianza mínima y pierden eficacia. Para verificar esta hipótesis nos fijamos en el gráfico de dispersión, el cuál debe presentar una buena dispersión de datos para que se cumpla la homocedasticidad, es decir, 
si observamos un cúmulo de datos recargado hacia la izquierda o derecha entonces hay heterocedasticidad.

\section{RESULTADOS Y DISCUSIÓN}

El procesamiento de los datos se realizó a través del programa estadístico SPSS 26, donde la confiabilidad del instrumento de medición utilizado para medir el bienestar psicológico en el trabajo presenta un coeficiente de Alfa de Cronbach de $=0.886$ y para el instrumento que mide el liderazgo transformacional presenta un Alfa de Cronbach de $=0.833 \mathrm{y}$ que según Hernández, Fernández y Bautista (2014) mientras más cercano a 1 se encuentre el Alpha de Cronbach tiene mayor confiabilidad.

El diseño del instrumento que se aplicó en la investigación comienza con los datos generales que incluye: género, edad, puesto de trabajo, antigüedad en la empresa y en el puesto, además del nivel de estudios.

De acuerdo con la recopilación de la información y la metodología utilizada en la investigación, se realizó el análisis de la regresión lineal simple para conocer cómo se comporta la variable dependiente representada por el bienestar psicológico en el trabajo a partir del comportamiento de la variable independiente representada por el liderazgo transformacional.

En la tabla 1, se muestra el resumen del modelo, donde se puede observar que el ajuste es bueno, ya que $R$ cuadrada ajustada $\left(R^{2} a\right)$ es 0.251 . Toma sentido basarnos en $R^{2}$ a para el ajuste, debido a que este coeficiente es $\mathrm{R}^{2}$ pero quitando el efecto de la variable independiente en el modelo. Así mismo, se pueden verificar dos supuestos; linealidad e independencia. Como $\mathrm{R}=0.503$, hay linealidad, pues la correlación es positiva y cercana a 1. Por su parte el coeficiente de Durbin-Watson es $=1.428$, cercano a 2 , el cual manifiesta que las observaciones muestrales son independientes cumpliendo con el supuesto de independencia.

\section{Tabla 1.- Resumen del modelob}

\begin{tabular}{|c|c|c|c|c|c|}
\hline Modelo & $\mathbf{R}$ & $\mathbf{R}$ cuadrado & $\begin{array}{c}\mathbf{R} \text { cuadrado } \\
\text { ajustado }\end{array}$ & $\begin{array}{l}\text { Error estándar de } \\
\text { la estimación }\end{array}$ & Durbin-Watson \\
\hline 1 & $.503^{\mathrm{a}}$ & .253 & .251 & .56430 & 1.428 \\
\hline
\end{tabular}

a. Predictores: (Constante), Liderazgo Transformacional

b. Variable dependiente: Bienestar Psicológico en el Trabajo

Fuente: Elaboración propia con información procesada en el programa estadístico SPSS $v 26$. 
En la tabla 2, denominada ANOVA se puede verificar la validez del modelo. Se observa que el p valor es cero, por lo cual el modelo es válido.

\section{Tabla 2.- ANOVA ${ }^{\mathrm{a}}$}

\begin{tabular}{llc|c|c|c|c}
\multicolumn{1}{c}{ Modelo } & $\begin{array}{c}\text { Suma de } \\
\text { cuadrados }\end{array}$ & gl & $\begin{array}{c}\text { Media } \\
\text { cuadrática }\end{array}$ & F & Sig. \\
\hline 1 & Regresión & 49.625 & 1 & 49.625 & 155.841 & $.000^{\mathrm{b}}$ \\
\hline Residuo & 146.478 & 460 & .318 & & \\
\hline Total & 196.102 & 461 & & & \\
\hline
\end{tabular}

a. Variable dependiente: Bienestar Psicológico en el Trabajo

b. Predictores: (Constante), Liderazgo Transformacional

Fuente: Elaboración propia con información procesada en el programa estadístico SPSS v26.

En la tabla 3, se muestra el coeficiente que permite obtener la ecuación de regresión, para ello se verifican los parámetros de $\mathrm{B}_{0}=2.699$ y $\mathrm{B}_{1}=.397$. Por lo tanto, se construye la siguiente ecuación:

Ecuación de regresión: $Y=2.699+.397 X$

Tabla 3.- Coeficientes ${ }^{a}$

\begin{tabular}{|c|c|c|c|c|c|c|}
\hline & Modelo & $\begin{array}{l}\text { Coe } \\
\text { esta }\end{array}$ & $\begin{array}{l}\text { entes no } \\
\text { arizados }\end{array}$ & $\begin{array}{c}\text { Coeficientes } \\
\text { estandarizados }\end{array}$ & $\mathbf{t}$ & Sig. \\
\hline & & B & Desv. Error & & & \\
\hline 1 & (Constante) & 2.699 & .132 & & 20.426 & .000 \\
\hline & $\begin{array}{l}\text { Liderazgo } \\
\text { Transformacional }\end{array}$ & .397 & .032 & .503 & 12.484 & .000 \\
\hline
\end{tabular}

a. Variable dependiente: Bienestar Psicológico en el Trabajo

Fuente: Elaboración propia con información procesada en el programa estadístico SPSS $v 26$.

En la figura 1, se muestra el histograma, donde se puede observar que las barras tienen un comportamiento similar a la curva normal, por lo cual si se cumple con el supuesto de normalidad. 


\section{Figura 1.- Histograma}

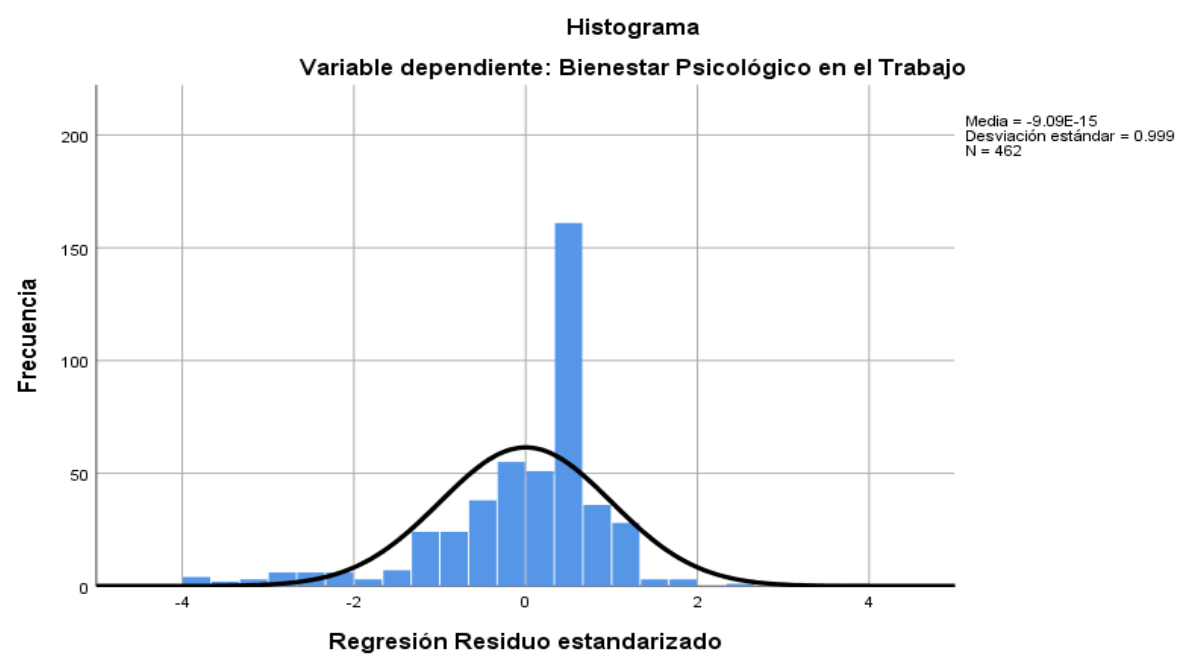

Fuente: Elaboración propia con información procesada en el programa estadístico SPSS $v 26$.

Para verificar este supuesto, puede observarse en la figura 2 el gráfico P-P que los datos se comportan como la línea recta, por lo tanto, se comprueba nuevamente que se cumple este supuesto.

\section{Figura 2.- Grafico P-P normal de regresión residuo estandarizado.}

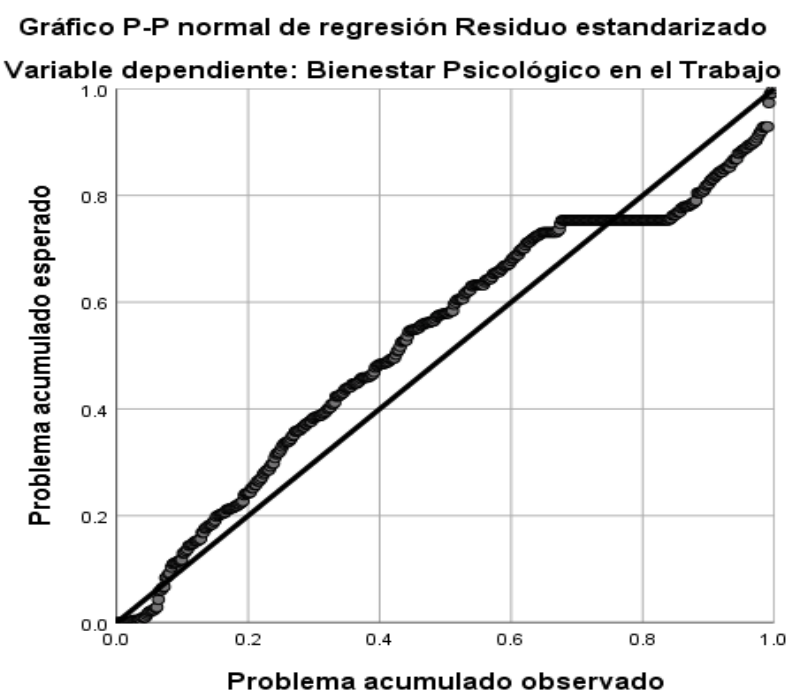

Fuente: Elaboración propia con información procesada en el programa estadístico

$$
\text { SPSS } v 26 .
$$


Finalmente, el diagrama de dispersión que se muestra en la Figura 3, verifica el supuesto de homocedasticidad. Se observa una buena distribución de los datos, no hay un cúmulo donde se concentre la mayoría de los datos, por lo que se acepta el supuesto de homocedasticidad.

\section{Figura 3-. Gráfico de dispersión.}

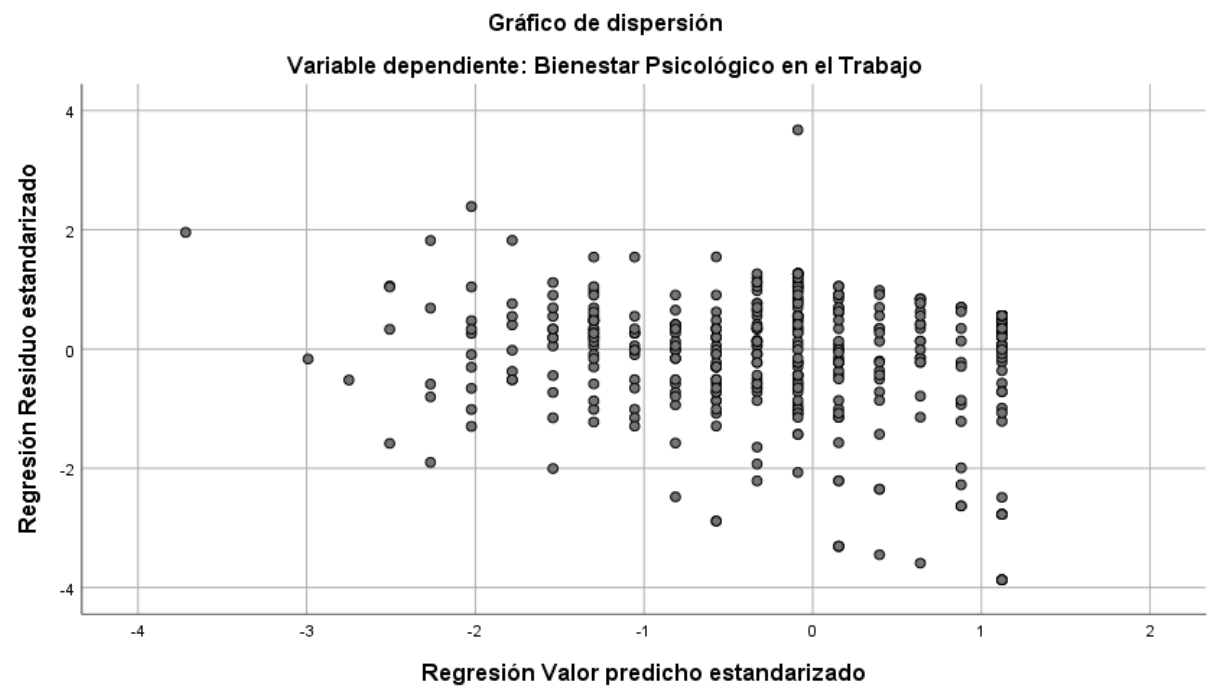

Fuente: Elaboración propia con información procesada en el programa estadístico

$$
\text { SPSS } v 26 .
$$

\section{CONCLUSIÓN O CONSIDERACIONES FINALES}

Se concluye que existe una relación positiva entre la variable liderazgo transformacional y la variable bienestar psicológico en el trabajo, ya que de acuerdo a los resultados se puede discernir que la puesta en práctica de un liderazgo transformacional generará un mejor bienestar psicológico en el trabajo. Derivado de lo anterior podemos afirmar que la hipótesis de trabajo planteada originalmente se acepta.

El modelo de regresión lineal simple propuesto, cumple con los supuestos mostrados, además el ajuste del modelo es bueno y con validez. Eso nos lleva a concluir que la medición del liderazgo transformacional y el bienestar psicológico en el trabajo en las MIPYMES de la ciudad de Durango México, es un factor de suma importancia para el mejor desempeño de las mismas.

Existe una correlación significativa entre el liderazgo transformacional y el bienestar psicológico en el trabajo desde la perspectiva de los trabajadores de las empresas estudiadas. El desarrollo del liderazgo transformacional dentro de las organizaciones incide de manera directa sobre el bienestar psicológico del trabajador quedando 
demostrado estadísticamente la existencia de una estrecha correlación entre ambas variables.

Los resultados del presente estudio permitirán que los gerentes y/o encargados de las 337 empresas estudiadas, tomen conciencia de lo importante que es poner en práctica un liderazgo transformacional en su organización para mantener o mejorar el bienestar psicológico en sus trabajadores, y de esta manera puedan llegar a marcar diferencia con otras organizaciones, además, les permitirá enfrentar los cambios que en la actualidad se exige a las empresas en el mundo globalizado, ya que no solamente se debe de estar preparado hacía el interior, sino también, hacía los factores externos que influyen en las organizaciones, ya sea de manera directa o indirecta y que al mismo tiempo representa un gran reto para el mercado.

Sin duda que el liderazgo transformacional bien aplicado puede generar una diferencia significativa no solo en la forma en la cual los trabajadores colaboran más allá de los límites que ellos mismos tenían pensado, pues como su nombre lo dice transforma su forma de pensar y de actuar cotidianamente en el trabajo al saberse tomados en cuenta por su líder, además se puede anotar la importancia del bienestar psicológico de los colaboradores como un motor importante en el logro de los objetivos organizacionales, de donde se desprende la importancia y la necesidad de generar organizaciones más humanas, donde se reconozca la valía del personal que ellas laboran.

\section{LISTA DE REFERENCIA.}

Avendaño, B. L., y Perrotini, I. (2015). Insuficiencia dinámica, crecimiento y desempleo en México, 1974-2012. Investigación Económica, LXXIV (293), 99-130. Recuperado de www.redalyc.org/articulo.oa?id=60141352006

Bass, B. M. (1985). Leadership and performance beyond expectations. New York, New York: Free Press.

Bolte, A., Goschke, T., y Kuhl, J. (2003). Emotion and intuition: effects of positive and negative mood on implicit judgments of semantic coherence. Psychological Science, 14, 416-421.

Carol, A. (2017). El liderazgo transformacional como herramienta en la gestión de equipos de trabajo. Universidad Militar Nueva Granada Facultad de Ciencias Económicas Especialización en Alta Gerencia, Bogotá D.C. 
Castro, A. (2010). Fundamentos de la Psicología Positiva. Buenos Aires, Argentina: Paidós.

Comisión Económica para América Latina y el Caribe. (2019). Evolución reciente del empleo en México. Recuperado de https://www.cepal.org/es/publicaciones/7477evolucion-reciente-empleo-mexico

Cook, J., y Wall, T. (1980). New work attitude measures of trust, organizational commitment and personal need non-fulfilment. Journal of Occupational Psychology, 53(1), 39-52.https://doi.org/10.1111/j.2044-8325.1980.tb00005.x

Cropanzano, R., y Wright, T. A. (2001). When a "happy" worker is really a "productive" worker: A review and further refinement of the happy-productive worker thesis. Consulting Psychology Journal: Practice and Research, 53(3), 182-199. https://doi.org/10.1037/1061-4087.53.3.182

Cújar, A., Ramos, C. D., Hernández, H. E., y López, J. M. (2014). Cultura organizacional: Evolución en la medición. Estudios Gerenciales. Universidad ICESI, 350-355.

D'raven, L., y Pasha-Zaidi, N. (2016). Using the PERMA Model in the United Arab Emirates. Social Indicators Research, 125(3), 905-933. Retrieved from https://link.springer.com/article/10.1007/s11205-015-0866-0

Dagenais-Desmarais, V., y Savoie, A. (2012). What is Psychological Well-Being, Really? AGrassroots Approach from the Organizational Sciences. Journal of Happiness Studies,13(4), 659-684. https://doi.org/10.1007/s10902-011-9285-3

Delfín, F., y Acosta, M. (2016). Importancia y análisis del desarrollo empresarial. Pensamiento \& Gestión, 40, 184-202. Obtenido de doi:dx.doi.org/10.14482/pege.40.8810

Deloitte. (2019). Séptima encuesta a Millennials y Gen Z. Recuperado de https://www2.deloitte.com/global/en/pages/aboutdeloitte/articles/millennialsurve y.ht ml?id=global:2em:3cc:4dcom_share:5awa:6dcom:about_deloitte

Devonish, D. (2013). Workplace bullying, employee performance and behaviors. EmployeeRelations, 35(6), 630-647. https://doi.org/10.1108/ER-01-2013-0004

Diener, E., y Biswas-Diener, R. (2002). Will money increase subjective well-being? Social Indicators $\quad$ Research, $\quad$ 57(2), 119-169. https://doi.org/10.1023/a:1014411319119 
Diener, E., y Seligman, M. (2004). Beyond money: toward an economy of well-

Ferrer, R. M. (2015). La influencia del factor humano, el liderazgo y la cultura de las organizaciones en los procesos de implementación y gestión del cambio organizacional. Rev. Int. Investig. Cienc. Soc., 11(1), 102-114.

García, M., Salas-Arbeláez, L., y Gaviria, É. (2017). Estilos de liderazgo de hombres y mujeres en las pymes. AD-minister. Universidad EAFIT, Medellín, Colombia, 31, $25-46$.

Great Place to Work. (2020). Atracción y Retención del Talento. Recuperado de https://www.greatplacetowork.com.mx/consultoria-de-cultura/atraer-y-retenertalento

Greenidge, D., Devonish, D., y Alleyne. (2014). The Relationship Between Ability-Based Emotional Intelligence and Contextual Performance and Counterproductive Work Behaviors: A Test of the Mediating Effects of Job Satisfaction. Human Performance,27(3), 225-242. https://doi.org/10.1080/08959285.2014.913591

Grupo Manpower. (2020). Resolviendo la escasez de talento: Crear, adquirir, tomar prestado y construir puentes (Encuesta de escasez de talento 2018).

Hernández, R., Fernández, C., y Baptista. (2014). Metodologia de La Investigacion (6th ed.). México, México: McGraw Hill.

Hindrichs, I., Juárez-García, A., Girardo, C., y Converso, D. (2016). Organizational Wellbeing in CSOs in Morelos (Mexico): Findings from a Qualitative Study. VOLUNTAS: International Journal of Voluntary and Nonprofit Organizations, 27(1),348-366. Retrieved from https://link.springer.com/article/10.1007/s11266015-9568- 0

Instituto Nacional de Geografía y Estadística. (2019). Encuesta Nacional sobre Productividad y Competitividad de las Micro, Pequeñas y Medianas Empresas (ENAPROCE). Recuperado 25 de septiembre, 2021 dehttp://www.inegi.org.mx/est/contenidos/proyectos/encuestas/establecimientos/ otras/enaproce/

Izquierdo, R., Novillo, L. A., y Mocha, J. V. (2017). El liderazgo en las microempresas familiares, desafíos y metas, Universidad y sociedad. Revista Científica de la Universidad de Cienfuegos, 9(3). 
Kuhl, J. (2000). A functional-design approach to motivation and self-regulation: the dynamics of personality systems interactions. En M. Boekaerts, R. Pintrich, y M. Zeidner (Eds.), Handbook of self-regulation (p. 111-169). San Diego, EUA: AcademicPress.

Lapo, M., y Jácome, M. J. (2015). El Liderazgo y su Evolución Histórica. Revista Empresarial ICE-FEE-UCSG, Ed. 36 9(4).

Medellín: Orbis.

Millán, R., y Castellanos, R. (2018). Bienestar subjetivo en México. México: Instituto de Investigaciones Sociales.

Morin, E. (1994). Introducción al pensamiento complejo (Rev. ed.). Barcelona: Gedisa.

Mosrin, A., Boudriás, J.-S., y Marsh, H. (2017). Complementary Variable and Person Centered Approaches. J Bus Psychol, 395-419.

Organización para la Cooperación y el Desarrollo Económico. (2020). Getting it Right:Prioridades estratégicas para México. Éditions OCDE,https://doi.org/10.1787/9789264292871-es

Peiró, J. M., Tordera, N., Lorente, L., Rodríguez, I., y Ayala, Y. (2014). Bienestar sostenibleen el trabajo: revisión y reformulación. Papeles Del Psicólogo, 35(1), 5-14. Retrievedfrom http://www.redalyc.org/articulo.oa?id=77830184002

Perilla, L. E., y Gómez, V. (2017). Relación del estilo de liderazgo transformacional con la salud y el bienestar del empleado: el rol mediador de la confianza en el líder. Revista de Psicología Del Trabajo y de Las Organizaciones, 33(2), 95-108.

Podsakoff, M., MacKenzie, S. B., Moorman, R. H., y Fetter, R. (1990). Transformational leader behaviors and their effects on followers\&\#039; trust in leader, satisfaction, andorganizational citizenship behaviors. The Leadership Quarterly, 1(2), 107142. https://doi.org/10.1016/1048-9843(90)90009-7

Rahman, S. A., Amran, A., Ahmad, N. H., y Taghizadeh, S. K. (2016). Enhancing the Wellbeing of Base of the Pyramid Entrepreneurs through Business Success: The Role of Private Organizations. Social Indicators Research, 127(1), 195-216. Retrieved fromhttps://link.springer.com/article/10.1007/s11205-015-0951-4

Reyes, L., López, R., y Reyes, A. (2016). Bienestar subjetivo en directivos de la Empresa deTransporte de Oriente. Encuentros, 89-103.

Seligman, M., Gillham, J., Reivich, K., y Linkins, M. (2009). Positive education: Positive 
psychology and classroom interventions. Oxford Review of Education, 35, 293311.

Stone, A. A., y Mackie, C. (2013). Subjective well-being: Measuring happiness, suffering, and other dimensions of experience. (Rev. ed.). Washington, DC, US: National Academies Press.

Teoh, K., Coyne, I., Devonish, D., Leather, y Zarola, A. (2016). The interaction between supportive and unsupportive manager behaviors on employee work attitudes. Personnel Review, 45(6), 1386-1402. https://doi.org/10.1108/PR-05-2015-0136

Valderrama, A. V. (2018). Liderazgo transformacional del director en la gestión educativaen una institución educativa pública. Universidad Cesar Vallejo, Lima.

Vázquez, L. (2018). El bienestar laboral como una ventaja competitiva en las organizaciones. 